\title{
Verificação multicritério das condições físicas para a segurança operacional aeroportuária
}

\author{
Ítalo Ponte Der Hovannessian Mota ${ }^{1}$, Francisco Heber Lacerda de Oliveira², Marcos Fábio Porto de Aguiar ${ }^{3}$
}

\begin{abstract}
Resumo: A garantia da segurança operacional aeroportuária é uma tarefa imprescindível, compulsória aos operadores de aeródromos e de difícil aplicação, por envolver diversos parâmetros técnicos, diferentes metodologias de avaliação e custos. $\mathrm{O}$ presente artigo objetiva analisar a razoabilidade dos resultados de uma pesquisa realizada com especialistas em infraestrutura aeroportuária a partir dos aspectos físicos de pistas de pousos e decolagens para a garantia da segurança das operações e a priorização de atividades de manutenção e reabilitação (M\&R). Considerando os parâmetros contidos na regulação nacional vigente, foram analisadas as condições de aderência, estruturais e funcionais por meio de uma Metodologia de Análise Hierárquica e o software Expert Choice, objetivando hierarquizar os critérios estudados. Os resultados da pesquisa mostram que as condições de aderência têm maior importância sobre as demais. Contudo, não devem ser aceitas como as únicas a serem priorizadas para a tomada de decisão sobre as estratégias de M\&R.
\end{abstract}

Palavras-chave: análise multicritério; manutenção; pavimentos; aeroportos.

Abstract: Airport operational safety assurance is an essential task, compulsory to operators of aerodromes and difficult to apply, because it involves many technical parameters, different asessment methodologies and costs. This paper aims to examine the reasonableness of the results of a survey conducted with experts in airport infrastructure about physical aspects of runways to assure the safety of operations and the prioritization of maintenance activities and rehabilitation (M\&R). Considering the parameters contained in the national regulation in effect, conditions of adherence, structural and functional were analyzed through a Analytic Hierarchy Process and software Expert Choice, in order to rank the studied criteria. The results show that the conditions of adherence have greater importance over the others. However, they should not be taken as the only ones to be prioritized for decision-making over M\&R strategies.

Keywords: multicriteria analysis; maintenance; pavements; airports.

\section{INTRODUÇÃO}

Visando uma melhor gestão para a infraestrutura aeroportuária, procurou-se fazer o reconhecimento das condições físicas do pavimento e suas influências para a segurança das operações. Assim, o presente artigo pretende tornar mais eficiente as tomadas de decisão, principalmente quando os gestores não possuírem todos os dados técnicos necessários para a alocação de recursos para atividades de manutenção e reabilitação (M\&R).

Foram empregados conhecimentos do Método de Análise Hierárquica - MAH (Analytic Hierarchy Process AHP), que é um método que auxilia as tomadas de decisão, para elaborar um modelo de priorização de condições, comparando as condições de aderência, com as condições estruturais e funcionais das pistas de pouso e decolagem. Para isso, foi feita uma pesquisa com especialistas na área, em busca do conhecimento deles a respeito das condições citadas.

\section{FUNDAMENTAÇÃO TEÓRICA}

$\mathrm{O}$ atrito é um dos parâmetros de maior importância sobre a condição física de um pavimento. Conforme Fernandes

\footnotetext{
1 Ítalo Ponte Der Hovannessian Mota, Departamento de Engenharia de Transportes, UFC. (italoponte@gmail.com)

2 Francisco Heber Lacerda de Oliveira, Centro de Ciências Tecnológicas, UNIFOR. (heberoliveira@unifor.br)

${ }^{3}$ Marcos Fábio Porto de Aguiar, Departamento de Construção Civil,

IFCE. (marcosfpa@gmail.com)
}

Manuscrito recebido em 10/11/2015 e aprovado para publicação em $30 / 05 / 2016$

Este artigo é parte de TRANSPORTES v. 24, n. 2, 2016. ISSN: 2237-1346 (online). DOI:10.4237/transportes.v24i2.1033
(2010, p. 158), este recebe uma maior atenção pelas entidades aeroportuárias, já que, os pavimentos das pistas devem proporcionar boa resistência à derrapagem em todos os tipos de condições climáticas. Sendo assim, os parâmetros que influenciam a resistência à derrapagem dos pavimentos molhados devem ser observados e quantificados, tais como: a profundidade de textura, acúmulo de borracha, o estado da sinalização diurna (marcas), depressões e desprendimento de material. Tendo em consideração que a inspeção visual não é suficiente para aferir estes parâmetros, devem-se programar ensaios que o façam. ANAC (2012, p. 3) proporciona ajuda na frequência e procedimentos a levar a cabo nas campanhas de medição de atrito.

Os sistemas de gerenciamento, segundo Causim (2001, p. 3), são instrumentos que permitem o planejamento adequado dos serviços de manutenção de um pavimento. A manutenção é necessária porque a contínua utilização do pavimento o predispõe ao aparecimento de defeitos superficiais, cujo reparo deve ser executado o mais cedo possível. O estado superficial de um pavimento flexível revela, em função do tipo de defeito visível e da sua frequência de ocorrência, o seu estado estrutural e/ou funcional. Dependendo do momento em que se pretenda desenvolver o processo de reabilitação do pavimento, tornase necessário o emprego de diferentes técnicas que resultam em diferentes graus de intervenção no pavimento existente.

Segundo a AASHTO (1997, p. 1), os sistemas de gerência de pavimentos incluem atividades e decisões relacionadas à concepção e à manutenção das vias. A implementação é considerada completa quando a gerência de pavimentos passa a fazer parte da rotina do processo de gestão, e a agência passa a utilizar a gerência de pavimentos 
Tabela 1. Escala Fundamental de números absolutos

Fonte: Saaty $(2008$, p. 4)

\begin{tabular}{c|c}
\hline Valor Atribuído (Aii) & Significado na Coluna \\
\hline 1 & Os elementos apresentam o mesmo grau de importância no alcance do objetivo \\
\hline 3 & Um elemento é um pouco mais importante do que o outro para o alcance do objetivo \\
\hline 5 & Um elemento é muito mais importante do que o outro para o alcance do objetivo \\
\hline 7 & Um elemento é muitíssimo mais importante do que o outro para o alcance do objetivo \\
\hline $2,4,6,8$ & Um elemento é extremamente mais importante do que o outro para o alcance do objetivo \\
\hline Valores de importância intermediária \\
\hline
\end{tabular}

para tomar decisões importantes, incluindo decisões de financiamento.

Conforme Haas et al (1994, p. 10), um Sistema de Gerência de Pavimentos fornece estratégias ótimas para tomadores de decisão, em todos os níveis gerenciais, derivadas completamente de procedimentos racionais claramente estabelecidos.

Um Sistema de Gerência de Pavimentos Aeroportuários - SGPA, na opinião de Fernandes (2010, p. 70), é essencialmente um sistema de informação. Como tal, o seu elemento de maior importância é a base de dados. Nela encontram-se reunidos os dados necessários à gestão da infraestrutura e ao seu estado num determinado momento.

De acordo com Shahin (1995, p 25), os benefícios a seguir foram enunciados por várias agências onde fora implementado SGPs: fornecer os dados necessários para os legisladores e gestores para determinação do orçamento; maximizar o retorno sobre o investimento do orçamento disponível para atividades de M\&R; criar um plano de priorizações de 5 anos; estabelecer os requisitos mínimos da condição; identificar as áreas que necessitam de manutenção; justificar projetos de M\&R; e critério para a distribuição do orçamento disponível entre várias redes.

Segundo a FAA (2006, p. 1), historicamente, a maioria das decisões de gestores de aeroportos a respeito das atividades de Manutenção e Reabilitação (M\&R), tem sido baseada na necessidade imediata ou na experiência, quando deveriam ser feitos planejamentos técnicos de longo prazo lastreados pela utilização de dados documentados. Essas abordagens não têm permitido os gestores de aeroportos evoluírem no quesito otimização de recursos em relação as estratégias de $M \& R$ por eles utilizadas.

Para Gomes (2001, p. 1), existe um campo do conhecimento chamado Pesquisa Operacional, concebido na época da Segunda Guerra Mundial. Dentro da Pesquisa Operacional, apareceram as metodologias de Apoio Multicritério à Decisão, na década de 70. Desde sua concepção, a Pesquisa Operacional serve para ajudar as pessoas a estruturar e analisar processos de tomada de decisão.

São diversos os métodos existentes que se utilizam das metodologias multicritério segundo Almeida (2006, p. 69). Na escola americana, os principais métodos são: Teoria da Utilidade Multiatributo - MAUT (Multi Atribute Utility Theory) e o Método de Análise Hierárquica - MAH (Analytic Hierarchy Process - AHP). Na escola francesa, os principais métodos são: ELECTRE (Elimination et Choix Traduisant la Réalité) e PROMÉTHÉÉ (Preference Ranking Organization Method for Enrichment Evaluations). Podem ainda ser citados os métodos: MACBETH (Measuring Attractiveness by a Categorical Based Evaluation Technique), TOPSIS (Tecnique for Order
Preference by Similarity to Ideal Solution), ANP (Analytic Network Process) e FDA (Fuzzy Decision Approach).

As metodologias de Apoio Multicritério à Decisão utilizam muitas ferramentas da psicologia quantitativa, que lida com processos cognitivos e escalas, para expressar a preferência com relação a aspectos totalmente subjetivos, segundo Saaty (1990, p. 9) e Gomes (2001, p. 1). Foram os estudiosos da psicologia quantitativa que descobriram que a melhor escala é aquela que termina em sete mais ou menos dois, ou seja, nove ou cinco, conforme descrito na Tabela 1. Com isso evitam-se as armadilhas comuns em questionários. A ancoragem - que é quando as notas ficam ancoradas em subintervalos da escala - é uma delas. Para quebrar esse efeito, quando se gera um formulário de pesquisa, aplica-se a ele escalas de um até cinco, sete ou até nove. Isso é conhecido pelos psicólogos desde os anos 50.

$\mathrm{O}$ maior benefício do MAH é a simplificação do problema em partes menores, que permite um maior domínio da situação como um todo, sintetizando a experiência e conhecimento do tomador de decisão através dos vetores de suas preferências e apontando a melhor alternativa segundo Nunes Junior (2006, p. 121). Esta vantagem deve ser respaldada por uma sólida experiência e conhecimento do tomador de decisão a respeito do problema decisório. O despreparo do tomador de decisão pode levar a uma hierarquia mal estruturada, indicando uma alternativa errônea como a melhor solução para o problema. Neste caso, a utilização de um especialista no assunto seria a ideal, pois a sua experiência seria imprescindível para viabilizar o MAH.

Segundo Nascimento (2010, p.15), o MAH organiza o problema de decisão em hierarquia, onde no primeiro nível está o objetivo global; seguido pelos critérios, no segundo nível; pelos subcritérios, no terceiro e pelas alternativas no último.

Os agentes tomadores de decisão possuem, geralmente, pontos de vista divergentes e diferentes juízos de valor, afirma Soares (2006, p. 127). Os métodos multicritérios de análise de decisão aparecem como uma ferramenta à gestão dos sistemas de transportes, onde as diversas variáveis envolvidas, um grande número de dados, interações e objetivos referentes a essas diversidades sejam avaliados de forma integrada, mostrando que a percepção da necessidade de mudanças pode ser atribuída a um processo de tomada de decisão, refletindo, de maneira suficientemente estável, o juízo de valores dos decisores.

\section{MÉTODOS, CRITÉRIOS, RESULTADOS E ANÁLISES}

No presente trabalho, seguiu-se a sequência estabelecida pelo MAH, que consiste em: estruturar o problema, definir e hierarquizar os critérios e indicadores, 


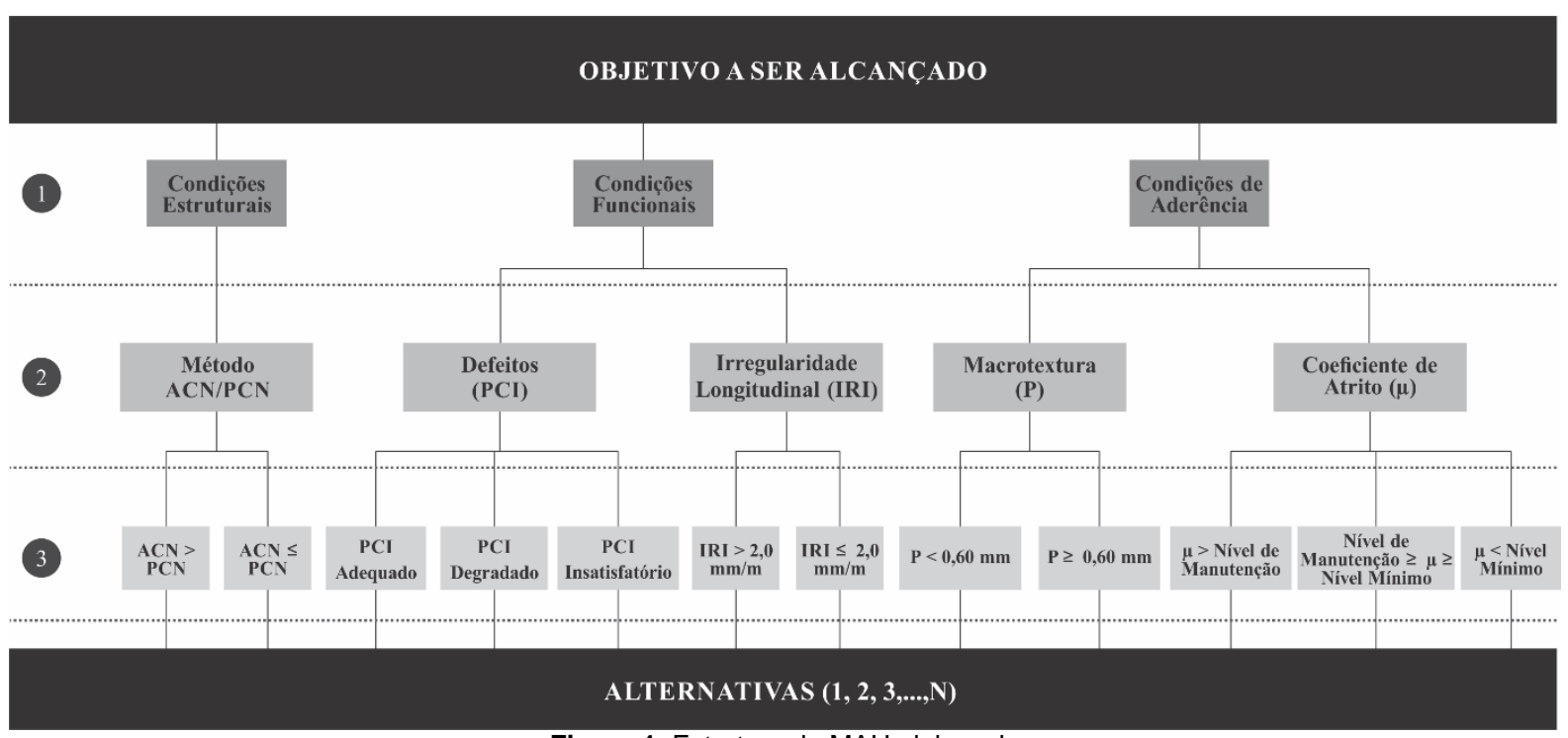

Figura 1. Estrutura de MAH elaborada

comparar par-a-par os critérios pertencentes a um mesmo grupo, calcular a consistência lógica, determinar as prioridades e analisar as alternativas. Para isso foi utilizando um software chamado Expert Choice com o objetivo de simplificar os cálculos, uma vez que este já os faz.

\subsection{Definição dos Critérios}

$\mathrm{Na}$ definição do MAH, verifica-se que a determinação dos critérios deve ser realizada a partir da opinião de especialistas, conforme Almeida (2006, p. 85). Para este trabalho foram levadas em conta considerações de Oliveira (2009) e acrescentados mais dois critérios de avalição que se referem também às condições superficiais de uma pista de pouso e decolagem. Estes critérios podem ser observados na Figura 1.

\subsection{Realização das comparações par a par}

Para a realização das comparações par a par, foi elaborado um questionário com base no modelo desenvolvido no trabalho de Almeida (2006, p. 72). Nesse questionário, estão presentes as comparações par-a-par entre os critérios de um mesmo grupo, os quais fornecem opções aos entrevistados para atribuir importâncias relativas entre os critérios. Também estão contidas no questionário as instruções para o correto preenchimento e uma avaliação onde o entrevistado pode deixar suas críticas, registrando a qualidade da estrutura de critérios adotada, em uma escala que vai de ótimo a ruim. O modelo de questionário exposto na Figura 2 foi enviado aos entrevistados, sendo registradas algumas críticas quanto à complexidade.

Então, foram inseridas explicações sobre os temas, concedendo maior compreensão da estrutura, dando uma nova forma de visualizar o questionário. A Figura 3 ilustra os itens inseridos no questionário.

Obteve-se o total de 24 entrevistas das quais 21 foram preenchidas por especialistas em aeroportos (operadores de aeródromos e do órgão regulador - Agência Nacional de Aviação Civil - ANAC) e 3 por professores da área de pavimentação e/ou aeroportos. Esses profissionais entrevistados ainda puderam contribuir com a estrutura do

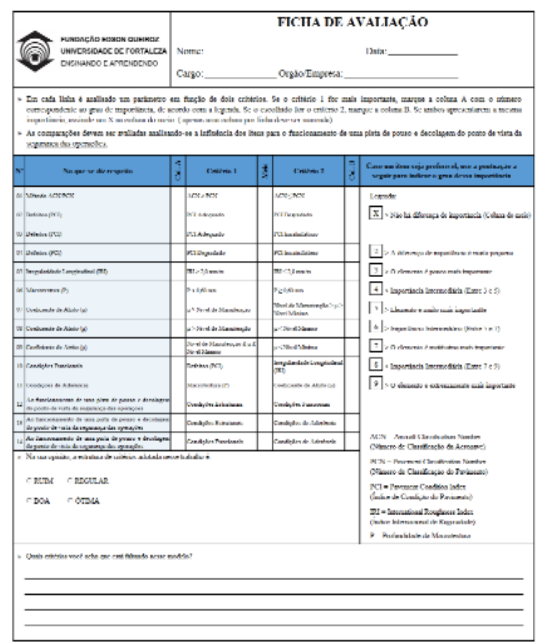

Figura 2. Primeira estrutura do questionário adotada Fonte: Adaptado de Oliveira (2014)

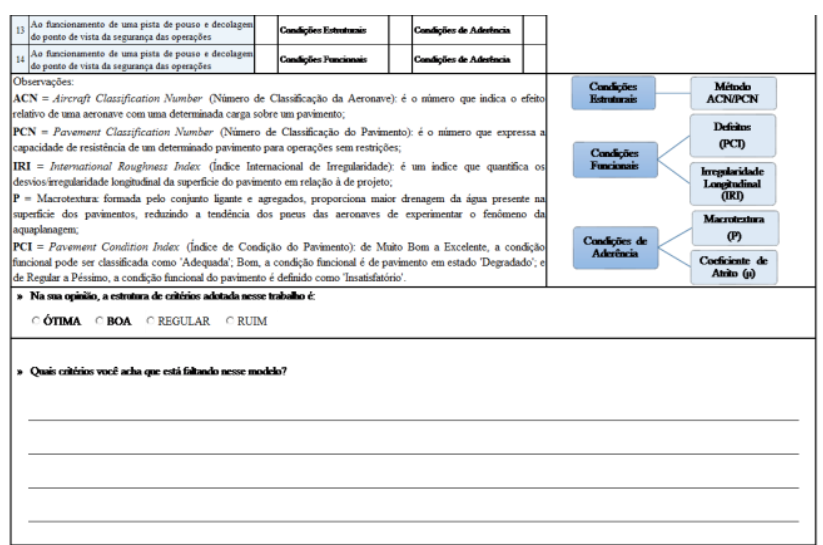

Figura 3. Nova estrutura do questionário

Fonte: Adaptado de Oliveira (2014)

formulário de pesquisa, e tiveram espaço para deixar sugestões e realizar críticas.

Essas sugestões e críticas, retiradas dos formulários a respeito dos critérios abordados e de sua estrutura, foram listadas a seguir:

a) Foi questionada a estrutura do questionário, que fora considerada óbvia em relação aos julgamentos a serem feitos nas comparações par a par; 
b) Houve sugestões para inserir a definição dos critérios;

c) Foi sugerido o acréscimo de critérios, como sinalização horizontal e vertical/luminosa;

d) Foi sugerido levar em consideração o sistema FOQA (Flight Operation Quality Assurance), uma vez que esse sistema auxilia o piloto dando informações das condições da pista durante as operações de pouso e a decolagem; e

e) Foi levantado o questionamento de um aspecto subjetivo, que seria a importância de o aeroporto possuir duas pistas de pouso de decolagem, favorecendo a segurança das operações em momentos de manutenção.

Vale ressaltar que parte das sugestões e críticas registradas pelos entrevistados, sobretudo o item (d), que mantém relação direta com as condições físicas das pistas e a segurança das operações de pousos e decolagens, deverão ser consideradas em momento posterior. Tais considerações são importantes para a continuidade dos estudos aqui iniciados e para melhoria contínua do processo de gerenciamento dos pavimentos aeroportuários e a tomada de decisão.

\subsection{Resultados Obtidos}

Após alimentar os questionários contidos no software Expert Choice individualmente para cada entrevistado, foi feita então a combinação desses valores gerando um só formulário. Todo esse processo ocorre de forma automática e ao final dele foram extraídas as informações dos pesos para cada critério de acordo com a Tabela 2.

Tabela 2. Pesos parciais gerados para cada critério

\begin{tabular}{c|c|c}
\hline \multirow{2}{*}{ Nível } & Critério & $\begin{array}{c}\text { Peso } \\
\text { Parcial }\end{array}$ \\
\hline $\mathbf{1}$ & Condições Estruturais & $\mathbf{0 , 1 1 3}$ \\
\hline $\mathbf{2}$ & Método ACN/PCN & $\mathbf{1 , 0 0 0}$ \\
\hline 3 & ACN $>$ PCN & 0,220 \\
\hline 3 & ACN $\leq$ PCN & 0,780 \\
\hline $\mathbf{1}$ & Condições Funcionais & $\mathbf{0 , 2 7 6}$ \\
\hline $\mathbf{2}$ & Defeitos $($ PCI) & $\mathbf{0 , 7 4 6}$ \\
\hline 3 & PCI Adequado & 0,733 \\
\hline 3 & PCI Degradado & 0,203 \\
\hline 3 & PCI Insatisfatório & 0,064 \\
\hline $\mathbf{2}$ & Irregularidade Longitudinal $($ IRI) & $\mathbf{0 , 2 5 4}$ \\
\hline 3 & IRI $>2,0$ mm/m & 0,153 \\
\hline 3 & IRI $\leq 2,0$ mm $/ \mathrm{m}$ & 0,847 \\
\hline $\mathbf{1}$ & Condições de Aderência & $\mathbf{0 , 6 1 2}$ \\
\hline $\mathbf{2}$ & Macrotextura $(\mathbf{P})$ & $\mathbf{0 , 3 3 0}$ \\
\hline 3 & P $<0,60 \mathrm{~mm}$ & 0,125 \\
\hline 3 & P $\geq 0,60 \mathrm{~mm}$ & 0,875 \\
\hline $\mathbf{2}$ & Coeficiente de Atrito $(\boldsymbol{\mu})$ & $\mathbf{0 , 6 7 0}$ \\
\hline 3 & $\mu>$ Nível de Manutenção & 0,727 \\
\hline 3 & Nível de Manutenção $\geq \mu \geq$ Nível Mínimo & 0,204 \\
\hline 3 & $\mu<$ Nível Mínimo & 0,069 \\
\hline
\end{tabular}

Os critérios do terceiro nível são os critérios mensuráveis para o alcance do objetivo. A Figura 4 mostra a saída de dados do software Expert Choice para esses critérios.

\subsection{Análise dos Resultados}

Com os resultados obtidos a partir da saída de dados do software Expert Choice, torna-se possível apontar qual dos critérios avaliados possui uma maior importância para manter a segurança das operações de pistas de pouso e decolagem, levando em consideração a opinião dos 24 entrevistados e os critérios estudados.

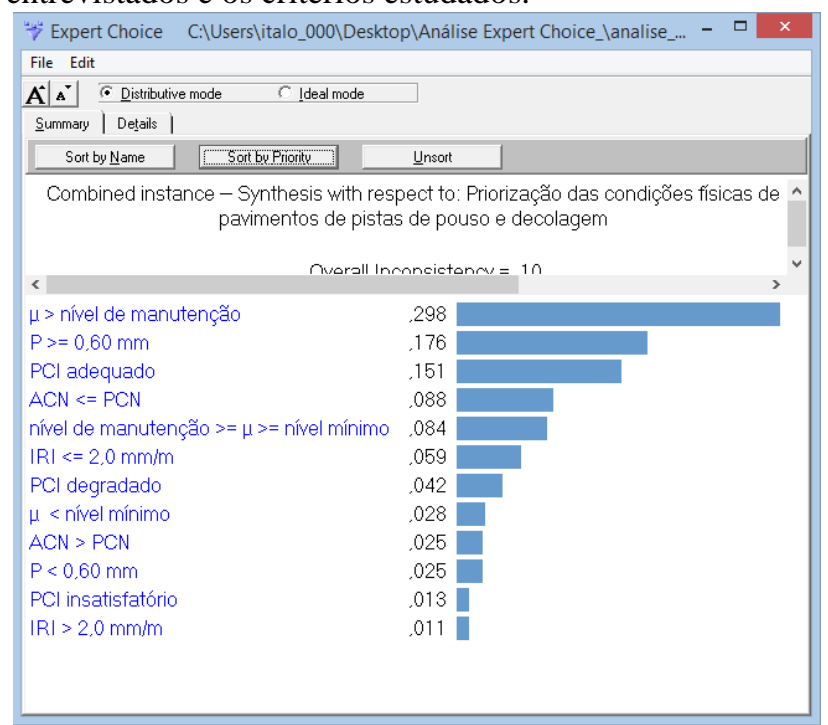

Figura 4. Saída de dados do software Expert Choice

Sendo assim foi elaborada a representação gráfica com o resultado e a porcentagem correspondentes a cada critério para uma melhor compreensão desses valores. Esta pode ser observada na Figura 5.
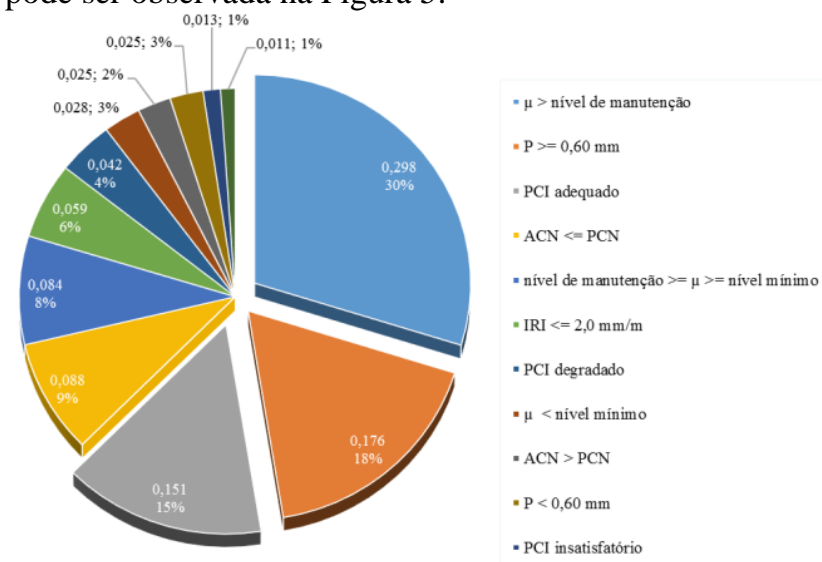

- PCI insatisfatório

: IRI $>2,0 \mathrm{~mm} / \mathrm{m}$

Figura 5. Análise gráfica dos critérios apresentados na Figura 4

Analisando os valores da Figura 5, pode-se concluir que de 12 critérios apresentados, dois deles, que seriam $\mu>$ nível de manutenção (30\%) e P > 0,60 mm (18\%), representam $48 \%$ do peso global de relevância quando se trata do funcionamento de uma pista de pouso e decolagem sob o ponto de vista da segurança das operações, de acordo com os 24 entrevistados. Essa representatividade pode, também, ser observada na Tabela 3 e na Figura 6, analisando o peso para as Condições de Aderência, grupo ao qual esses dois critérios estão contidos.

Com os dados analisados pode-se então priorizar os critérios do primeiro nível em ordem decrescente, afirmando novamente que para aquele grupo seleto de profissionais entrevistados, os critérios analisados e 
levando em consideração a segurança das operações em pistas de pouso e decolagem, tem-se então que Condições de Aderência > Condições Funcionais > Condições Estruturais, em grau de importância.

Os resultados apresentados parecem ser razoáveis, pois traduzem uma questão de prática contínua entre as equipes técnicas dos operadores de aeródromos e inserida nos procedimentos de manutenção das pistas de pouso e decolagem dos aeroportos brasileiros há, pelo menos, duas décadas. A macrotextura e o coeficiente de atrito, inseridos na Condição de Aderência, sempre foram (e continuam sendo) parâmetros de cumprimento obrigatório por parte do operador de aeródromo e de fiscalização dos órgãos reguladores (o Departamento de Aviação Civil - DAC, até 2005, e a Agência Nacional de Aviação Civil - ANAC, a partir daquele ano).

\begin{tabular}{cccc}
\multicolumn{4}{c}{ Tabela 3. Critérios do primeiro nível } \\
\hline $\begin{array}{c}\text { Grau de } \\
\text { Importância }\end{array}$ & Critério & Peso Parcial & $\%$ \\
\hline 1 & Condições de Aderência & 0,612 & 61 \\
2 & Condições Funcionais & 0,276 & 28 \\
3 & Condições Estruturais & 0,113 & 11 \\
\hline
\end{tabular}

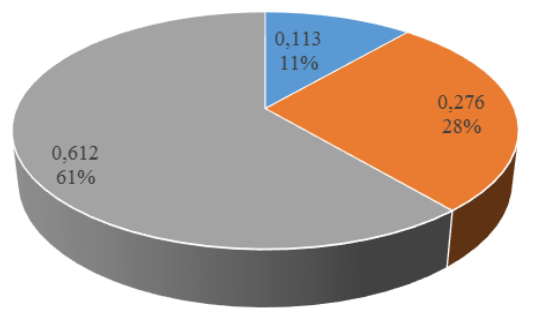

- Condições Estruturais - Condições Funcionais = Condições de Aderência

Figura 6. Representatividade dos critérios do primeiro nível

O ACN e o PCN, por sua vez, são de caráter obrigatório nos projetos de implantação de infraestruturas de pátios e pistas aeroportuários. Após construídas, essas infraestruturas têm suas condições estruturais avaliadas somente quando existe alguma demanda extraordinária por parte do órgão regulador ou suspeita do operador de aeródromo de comprometimento estrutural, identificados por meio de vistorias que detectam afundamentos, rupturas ou colapsos.

Os defeitos superficiais e a irregularidade longitudinal começaram a ser verificados in situ e com frequências definidas, como de caráter compulsório aos operadores de aeródromos, somente a partir de 2013, ou seja, algo muito recente na realidade dos aeroportos brasileiros. Vale registrar que as análises das condições funcionais eram feitas pelos operadores de aeródromos, até o estabelecimento da regulação nacional em 2012, de modo eventual e sem metodologia especificada.

Percebe-se, portanto, que o fato de as condições de aderência terem uma maior relevância e um maior tempo de aplicação no cenário aeroportuário nacional, contribuiu para que mais da metade dos especialistas entrevistados indicassem essas condições como as mais importantes, notadamente, para a gerência de pistas de pouso e decolagem já em operação. A preocupação, nestes casos, é manter as condições de aderência dentro dos padrões de segurança recomendados.
Os autores entendem a relevância das condições de aderência para a segurança das operações de pousos e decolagens, mas defendem que essas condições estão, de muitos modos, interligadas às demais. Não se concebe o funcionamento satisfatório e abrangente de um complexo de pátios e pistas aeroportuários sem que todas as condições analisadas neste trabalho estejam atuando sem restrições às operações.

É notório que, se a capacidade de suporte da infraestrutura da pista é insuficiente para operação de determinadas categorias de aeronaves, consequentemente, as operações de pousos e decolagens ficam comprometidas; se há presença de defeitos na pista ou se há irregularidades de ordem funcional, também as operações ficam afetadas.

Em todos os casos, o operador de aeródromo deve estar em contínua atenção à prevenção dos incidentes e acidentes por meio da manutenção preventiva e corretiva de todas as condições expostas, sejam elas de aderência, funcionais ou estruturais, objetivando salvaguardar o patrimônio e, principalmente, as vidas que estão sob sua responsabilidade.

\section{CONCLUSÕES}

Neste trabalho foram analisadas as condições físicas de pavimentos aeroportuários utilizando o Método de Análise Hierárquica, e teve como objetivo priorizar estas em relação à segurança das operações em pistas de pouso e decolagem. Para isso, estas condições foram divididas em condições estruturais, funcionais e de aderência.

Os resultados encontrados simularam um cenário em que as condições de aderência foram consideradas as mais importantes, sendo as condições funcionais as de segunda maior importância e, por último, as estruturais. Tais resultados não devem ser interpretados como indicativo único, obrigatório e prioritário de manutenções e reabilitações a serem executadas pelos operadores de aeródromos para as condições de aderência (macrotextura e o coeficiente de atrito). Deve-se analisar o estado global das condições físicas das pistas de pousos e decolagens e o comprometimento desse estado para a segurança das operações. Isso para que se tenha uma indicação aceitável da estratégia de M\&R mais provável que solucione ou mitigue os problemas, proporcionado os maiores benefícios com os menores custos, e, por consequência, aperfeiçoando o processo de tomada de decisão. Vale reiterar que os resultados apresentados neste trabalho são considerados válidos apenas para as respostas dos 24 profissionais entrevistados e os critérios selecionados.

\section{REFERÊNCIAS}

Agência Nacional de Aviação Civil (2012). Resolução ํo 236: de 5 de Junho de 2012. Brasília: ANAC. 19 p.

Almeida, R. V. de O. (2006) Concepção de Modelos de Avaliação de Condições de Rolamento e Indicação de Priorização de Vias como Etapas de um Sistema de Gerência de Vias não Pavimentadas. 195 f. Dissertação (Mestrado) - Curso de Programa de Mestrado em Engenharia de Transportes, Centro de Tecnologia, Universidade Federal do Ceará, Fortaleza.

American Association of State Highway and Transportation Officials. (1997) E1889-97: Standard Guide for Pavement 
Management Implementation. Pennsylvania: Astm International. $4 \mathrm{p}$.

Causim, P. B. (2001) Estudo de um Sistema de Gerencia de Pavimentos para Cidades de Pequeno e Médio Porte. 2001. 128

f. Dissertação (Mestrado) - Curso de Engenharia Civil,

Universidade Estadual de Campinas, Campinas.

Federal Aviation Administration. (2006) Advisory Circular 150/5380-7A: Airport Pavement Management Program. Washington: U.s. Department Of Transportation. 15 p.

Fernandes, C. I. C. dos S. H. (2010) Sistemas de Gestão de Pavimentos Aeroportuários Caracterização e Aplicabilidade: Caracterização e Aplicabilidade. 173 f. Dissertação (Mestrado) Curso de Engenharia Civil, Instituto Superior Técnico, Lisboa.

Gomes, L. F. A. M. (2001) Tomadas de Decisão são Facilitadas com Modelos Matemáticos. 2001. Disponível em:

<http://www.comciencia.br/entrevistas/modelagem/autran.htm>. Acesso em 18 out. 2014.

Haas, R.; Hudson W. R.; Zaniewski, J. (1994), Modern Pavement Management. Krieger Publishing Company, Malabar, Florida, 1994

Nascimento, L. P. A. da S. (2010) Aplicação do Método AHP com as Abordagens Ratings e BOCR: Projeto F-X2. $150 \mathrm{f}$. Tese (Doutorado) - Curso de Engenharia Aeronáutica e Mecânica, Instituto Tecnológico de Aeronáutica, São José dos Campos.

Nunes Junior, L. F. (2006) Tomada de Decisão com Múltiplos Critérios: Pesquisa-Ação sobre o Método AHP em Pequenas Empresas. 126 f. Dissertação (Mestrado) - Curso de Contabilidade e Administração, Departamento de Economia, Universidade de Taubaté, Taubaté.

Oliveira, F. H. L. de. (2009) Proposição de Estratégias de Manutenção de Pavimentos Aeroportuários Baseadas na Macrotextura e no Atrito: Estudo de Caso do Aeroporto Internacional de Fortaleza. 2009. 203 f. Dissertação (Mestrado) - Departamento de Engenharia de Transportes, Universidade Federal do Ceará, Fortaleza.

Oliveira, F. H. L. de. (2014) Exame de Qualificação da Tese de Doutorado. Programa de Pós-Graduação em Engenharia de Transportes. Departamento em Engenharia de Transportes. Centro de Tecnologia. Universidade Federal do Ceará, Fortaleza.

Saaty, T. L. (2008) Decision making with the analytic hierarchy process. International Journal of Services Sciences. Genebra, p. 83-98. DOI: 10.1504/IJSSCI.2008.017590

Saaty, T. L. (1990) How to make a decision: The Analytic Hyerarchy Process. European Journal of Operational Research. North-Holland, p. 9-26. DOI: 10.1287/inte.24.6.19

Shahin, M. y. (1995) Pavement Management for Airports, Roads, and Parking Lots. London. Chapman \& Hall. 450 p.

Soares, U. P. (2006) Procedimento para a Localização de Terminais Rodoviários Interurbanos, Interestaduais e Internacionais de Passageiros. 343 f. Dissertação (Mestrado) Curso de Mestrado em Engenharia de Transportes, Universidade Federal do Rio de Janeiro, Rio de Janeiro. 